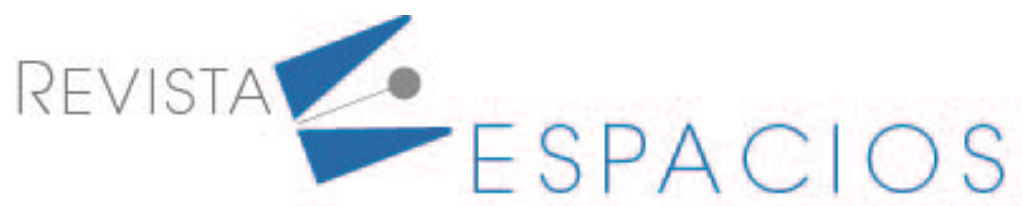

\title{
Comercio electrónico y estrategia de empresa a empresa (B2B): una revisión bibliométrica
}

\section{Electronic commerce and business to business strategy: a bibliometric review}

\author{
ALVAREZ-AROS, Erick L. ${ }^{1}$ \\ BARRAGÁN, José L. ${ }^{2}$ \\ MENÉNDEZ, Jorge $\mathrm{E}^{3}$

\section{Resumen} \\ Este trabajo presenta un análisis holístico del comercio electrónico y la modalidad Negocio a Negocio \\ (B2B) en la estrategia organizacional. Para ello, se realizó un análisis bibliométrico en las plataformas \\ Web of Science y Scopus. Entre los resultados destaca que pocos estudios abordan conjuntamente al \\ comercio electrónico con la modalidad negocio a negocio; China, Malasia y Canadá son los países con \\ mayor producción de artículos sobre B2B y tlos emas de tendencia frecuente fueron social media, \\ confianza e innovación.
}

Palabras clave: análisis bibliométrico, biblioshiny, b2b, comercio electrónico

\begin{abstract}
This paper presents a holistic analysis of electronic commerce and Business-to-Business (B2B) modality in organizational strategy. For this, a bibliometric analysis was performed on the Web of Science and Scopus platforms. Among the results, it stands out that few studies jointly address electronic commerce with the business-to-business modality; China, Malaysia and Canada are the countries with the highest production of articles on $\mathrm{B} 2 \mathrm{~B}$, and the most common trends were social media, trust and innovation.
\end{abstract}

Key words: bibliometric analysis, biblioshiny, b2b, e-commerce

\section{Introducción}

La llegada del internet cambió radicalmente la manera de hacer negocios. En este sentido, los medios digitales ofrecen alternativas diferentes a los métodos convencionales para que las empresas comercialicen sus productos y servicios. De esta forma conceptos como el comercio electrónico (e-commerce o EC) han surgido en los mercados hasta el grado de ser un elemento impredecible para negociar estratégicamente en organizaciones de diferentes giros y sectores, en las cuales se garantiza la transmisión segura de información y los fondos, y se elimina la presencialidad de actores y espacios físicos para negociar (Chandrasekar Subramaniam, 2002; Laudon \& Traver, 2016; Tsai \& Chiang, 2011).

\footnotetext{
${ }^{1}$ Universidad Popular Autonoma del Estado de Puebla. México. Email: erickleobardo.alvarez@upaep.mx

2 Universidad Popular Autonoma del Estado de Puebla. México. Email: joseluis.barragan@upaep.edu.mx

${ }^{3}$ Universidad Popular Autonoma del Estado de Puebla. México. Email: jorgeemanuel.menendez@upaep.edu.mx
} 
Cabe aclarar que el e-commerce es aquel en el cual se intercambian bienes y servicios por medios electrónicos y se relaciona con actividades como la comunicación de información, gestión de pagos, instrumentos financieros y transportación. De esta forma el negocio electrónico permite alinear los objetivos empresariales a la estrategia y facilita la creación de nuevos productos, mercados, canales de distribución y reduce el costo de las actividades empresariales (Chan, 2004; Fernández-Portillo, Sánchez-Escobedo \& Jiménez-Naranjo, 2015; Heng, 2003; Moreno, Sánchez, Moreno \& de Pablos Heredero, 2016).

En este sentido, el e-commerce de Negocio a Negocio (B2B) es una modalidad muy usada e implica la negociación entre el vendedor y comprador, por lo tanto, el B2B es el soporte electrónico de transacciones comerciales entre compañías que permite a una empresa establecer relaciones electrónicas con sus socios. En este sentido, el ecommerce continúa creciendo y en el futuro será la más amplia forma de negociación organizacional. Por lo tanto, este trabajo exploró holísticamente la evolución del e-commerce dentro de la estrategia organizacional y su relación con el modelo de negocio B2B a través de un análisis bibliométrico (Fensel, Ding, Omelayenko, Schulten, Botquin, Brown \& Flett, 2001; Martínez-López, Merigó, Valenzuela-Fernández \& Nicolás, 2018; Tomak \& Xia, 2002; Zhu, Li, Zhang \& Shi, 2017).

Para dicho propósito se realizó un estudio bibliométrico como en otras investigaciones (Carlsson, Larsson, Svensson \& Åström, 2017; Mou, Cui \& Kurcz, 2019; Muhuri, Shukla \& Abraham, 2019). Dicho análisis fue realizado a través del software estadístico R, Bibliometrix y Biblioshiny, entre el periodo 2010 al 2018 en las bases de datos Web of Science (WoS) y Scopus, y se obtuvo información de carácter cuantitativo sobre la producción científica del tema, las revistas más significativas, los autores más destacados y los países con mayor producción científica del tema entre otros. Este estudio se conforma de la presente introducción, posteriormente se presenta el marco teórico, la metodología, los resultados y discusión, y las conclusiones.

\subsection{El e-commerce y la estrategia B2B}

La evolución y divulgación del e-commerce se remonta al periodo donde se crearon las primeras supercomputadoras y el comercio en línea, a finales de la década de los 60's, con el uso de tecnologías como el intercambio electrónico de datos y la transferencia de fondos electrónicos por parte de empresas como Ford y General Motors, las cuales observaron las dificultades de costos, velocidad y certeza residentes en las transacciones sobre papel (Eastin, 2002; Kiriakidis, 2016; Tuunainen, 1998; Williams \& Frolick, 2001).

En sus inicios el e-commerce afrontó problemáticas relacionadas a los temas de comunicación, del proceso de negocios entre los actores comerciales, de servicios ofrecidos y del contexto de los procesos no presenciales o en línea; además, esto implicó mejorar la experiencia de los consumidores, las empresas, el gobierno y los dispositivos móviles. En el proceso también se resolvieron problemáticas como las relacionadas con la falta de trato personal entre el cliente y el comprador, la incapacidad de experimentar el producto antes de comprarlo, la necesidad de acceso a internet para realizar la transacción, fraudes, problemas de seguridad, atrasos logísticos, impuestos aduaneros, devolución de productos, desarrollo de infraestructura para el comercio electrónico entre otros (Alrawi \& Sabry, 2009; Damanpour \& Damanpour, 2001; Niranjanamurthy et al., 2013).

Además de lo anterior, el termino en sí del comercio electrónico ha sufrido diversas transformaciones y enfoques según distintos autores, que van desde enfoques logísticos y relacionados a la cadena de suministros hasta enfoques hacia diferentes interacciones y usuarios (Monroe \& Barrett, 2019). Por ejemplo, autores muy citados, como Sila (2013) y Brodie (2000a, 2000b), plantean un concepto unitario del B2B EC, definido como todas las tecnologías B2B habilitadas para Internet que permiten a los socios de la cadena de suministro comprar y vender productos y servicios y compartir información. 
Dichos autores usan este término indistintamente con frases como sistemas interorganizacionales basados en internet, negocio electrónico, comercio electrónico y tecnologías web, siempre que impliquen transacciones entre empresas. Dos años después, el autor continúa con el mismo concepto de B2B EC orientado a la cadena de suministros en el mercado europeo; el artículo tiene un coautor de origen canadiense que seguramente aporta conocimiento geográfico.

Chen (2013) define el e-commerce con un enfoque hacia la logística, propone que el comercio electrónico ofrece la oportunidad de intercambiar información y facilita un mayor poder adquisitivo de clientes dispersos. Así mismo define B2B con un enfoque a herramientas como ERP (Sistema Planeador de Recursos Empresariales por sus siglas en ingles) y CRM (Administración basada en relaciones con los clientes por sus siglas en ingles), y B2B construyen y facilitan las relaciones entre diferentes entidades comerciales que operan dentro de una cadena de suministro.

Desde otra perspectiva, Hsu y Chiang (2017) unen los dos conceptos en uno, B2B EC, con un enfoque orientado a la lealtad de los clientes, mencionando que la entrega inmediata de contenido con valor agregado, la interacción con los clientes y el aumento de la información compartida entre los usuarios, se han convertido en sus características más competitivas.

Por su parte, Zhang (2017) define el e-commerce como el sistema de comercio logístico que considera la colaboración entre diferentes proveedores de servicios para optimizar el beneficio total de la alianza. El B2B es definido como un acuerdo comercial entre compañías que operan al mismo nivel en la cadena de suministro para lograr un objetivo común.

De los conceptos anteriores se puede argumentar que el termino e-commerce mantiene una variedad de enfoques que le permiten relacionarlo desde diferentes perspectivas en los ámbitos comerciales y tecnológicos, y es precisamente en este último ámbito donde ha ocurrido una importante evolución del tema (Dai, \& Kauffman, 2002; Wilkinson, 2006).

Del punto anterior, en cuanto a otras tecnologías necesarias para formalizar el comercio electrónico, destacan los beneficios más relevantes del intercambio electrónico de datos empresariales, se encuentra una mejor relación cliente-proveedor, una mayor precisión en los pedidos, una reducción de costos, ahorro de tiempo, mejor manejo de las facturas y las notificaciones de que algunos de los productos no están disponibles. Aunado a lo anterior, con la llegada del internet se acrecentó el interés de las empresas en incorporar la manera de hacer negocios a una conectividad universal entre pequeñas, medianas y grandes empresas por la posibilidad de contar con millones de consumidores potenciales (Breen \& Crawford, 2005; Oliveira, Zhang \& Zhang, 2007; Wigand, 1997).

Con todo lo anterior, las innovaciones, la tecnología y el comercio electrónico generaron diferentes vertientes como el e-commerce, clasificado dentro de actividades como el envío de información de productos y servicios, pagos por teléfono o redes informáticas u otro medio de comunicación, la aplicación tecnológica de automatización en las transacciones comerciales, las herramientas de reducción de los costos de servicios, la calidad de los bienes, el aumento de la velocidad de los servicios de entrega y la capacidad de comprar y vender productos por internet y otros servicios en línea (Blake, 2001; Klopping \& McKinney, 2004; Marín-Idárraga \& Cuartas-Marín, 2019; Xiao \& Dong, 2015).

Es por ello que en la actualidad la tendencia de las empresas es expandirse al mercado electrónico, añadiendo roles empresariales con un enfoque digital y permitiendo mejorar el desempeño organizacional a través de la innovación. De este modo, la aparición de nuevos competidores y consumidores cada vez más informados subraya la necesidad que tienen las organizaciones de contar con información de calidad que les permita 
enfrentar los desafíos de este nuevo escenario electrónico (Fonseca, 2014; Jones, Motta \& Alderete, 2016; Kiron, Kane, Palmer, Phillips \& Buckley, 2016; Thatcher, Foster \& Zhu, 2006).

De esta manera surgieron diferentes formas de comercio electrónico, entre las cuales se involucra a las interacciones digitales entre organizaciones, gobierno, consumidores, ciudadanos, comercio móvil y sus interacciones. Sin embargo, para este estudio en particular, se definió como objetivo analizar holísticamente el e-commerce entre empresas, por ser este último, elemento fundamental para el desempeño empresarial, pues en este nuevo tipo de comercialización se descarta la manera presencial de interaccionar y la necesidad de usar espacios físicos (Burt \& Sparks, 2003; Kumar, Sharma, Vatavwala \& Kumar, 2019; Ngai \& Wat, 2002).

\section{Metodología}

La metodología utilizada en el presente proyecto fue la técnica del análisis bibliométrico, definida como la disciplina instrumental que cuantifica las propiedades de la literatura científica a fin de valorar la actividad académica y científica en diferentes campos de la ciencia. Consiste en la aplicación de métodos tanto estadísticos como matemáticos para presentar los avances del conocimiento humano mediante la cuantificación de documentos (Becerra \& Fleitas, 2002; Moreno, Sánchez, Moreno \& de Pablos Heredero, 2016; Hew, 2017).

Para estudiar holísticamente e-commerce en la modalidad B2B se realizó un análisis bibliométrico de documentos recolectados de las bases de datos WoS y Scopus. Dicha búsqueda fue filtranda en al área del título para los términos "e-commerce" y "B2B". La búsqueda fue limitada a publicaciones desde el 2010, un año previo en que se refleja el crecimiento de dichas publicaciones en WoS, hasta el 2018, por ser este un año con publicaciones completas previo a la fecha de recopilación de documentos (Fernandez, Nicolas \& Merigó, 2019; Geng, Wang, Zuo, Zhou, Du \& Mao, 2017; Valenzuela, Merigó, Johnston, Nicolas \& Jaramillo, 2017).

El proceso de recopilación de artículos se realizó el 23 de diciembre del 2019 en las plataformas WoS y Scopus, de dicha búsqueda se obtuvieron 981 resultados del término "e-commerce" de WoS, 422 documentos del término "B2B" de WoS y 22 publicaciones del término "e-commerce" en conjunto con el término "B2B" de WoS. Del mismo modo, se obtuvieron 4,978 resultados de Scopus del término "e-commerce", 902 documentos del del término "B2B" de Scopus y 150 publicaciones del término "e-commerce" en conjunto con el término "B2B" en la base de datos Scopus.

Una vez descargado el archivo en formato ".bib" o ".txt" de las bases de datos WoS y Scopus que contiene la información bibliométrica, este se procesa en el software bibliométrico en tres procesos. La Figura 1 explica el flujo de trabajo de dichos procesos realizado por el software Bibliometrix y Biblioshiny:

Figura 1

Flujo de trabajo para análisis bibliométrico

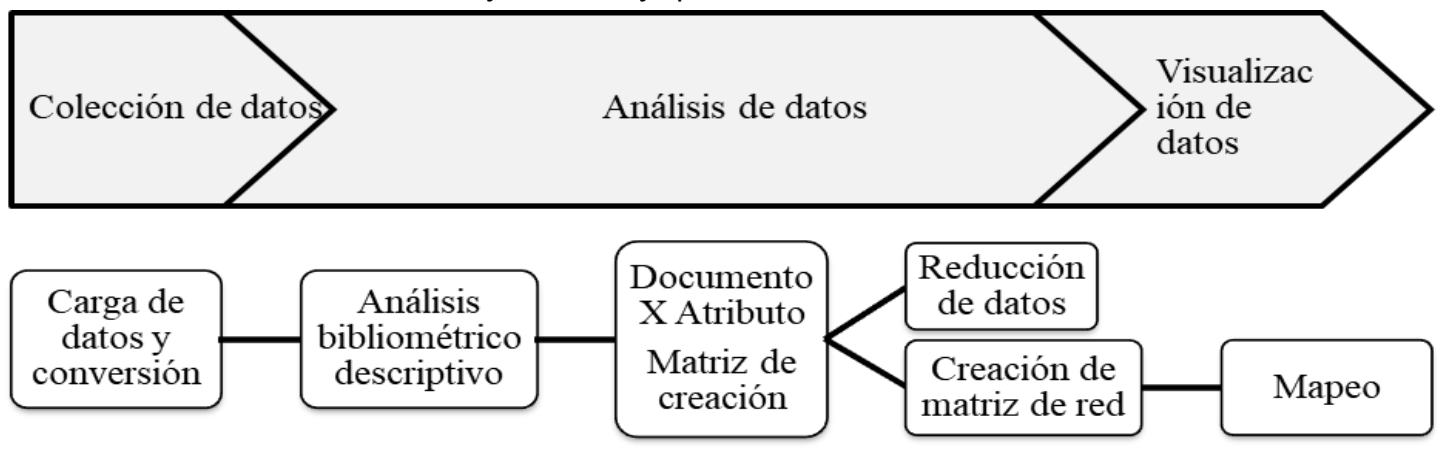

Fuente: Software R, Bibliometrix y Biblioshiny (2019) 
De la Figura 1 se observa que el software procesa el trabajo en tres fases, en la primera fase procesa la colección de datos, es decir, el conjunto de información descargada, tanto de WoS como de Scopus, se debe cargar en la página de Biblioshiny para proceder a su conversión. En la segunda fase, se hace primeramente un análisis bibliométrico descriptivo, posteriormente, se relacionan los documentos con sus atributos para crear una matriz y normalizar la información, seguidamente, el software separa la información en dos secciones, la primera sección enfocada a la reducción de datos y la segunda sección, enfocada a la creación de matrices de red, pasando a su vez por un proceso de normalización. Por último, en la tercera fase se realiza el proceso de visualización de datos que reúne tanto los datos reducidos como la creación de matrices de redes para mapearlas y visualizar dicha información (Li, Sheng \& Ma, 2018; Villa, Ruiz, Valencia \& Picón, 2018; Zadykowicz, Gębarowski \& Siemieniako, 2019).

Del punto anterior es importante aclarar que el análisis bibliométrico realizado está basado en dos tipos de indicadores: de relación y de actividad. Los indicadores de actividad contienen información relacionada con la cantidad y el impacto de los artículos publicados. Además, una novedad realizada en este estudio fue que los resultados también se estudiaron mediante índices internacionales como el índice $\mathrm{G}$, y se muestra información relativa al número de documentos por autor, colaboración entre países, producción de documentos por país, documentos más relevantes, entre otros. (Moreno, Sánchez, Moreno \& de Pablos Heredero, 2016; Wang, Chen, Rogers, Ellram \& Grawe, 2017).

Por otra parte, los indicadores de relación son utilizados para trazar las relaciones e interacciones entre los artículos estudiados. En este sentido, se compararon las variables en cuanto a su comportamiento, relación y diferencias de su evolución a lo largo del periodo estudiado (Donthu, Kumar \& Pattnaik, 2020; Merigó \& Yang, 2017; Roig-Tierno, Gonzalez-Cruz \& Llopis-Martinez, 2017).

Por último, para concluir con el análisis bibliométrico que proporcionó una visión holística y redujo subjetividad en el proceso de análisis entre los documentos encontrados del B2B y el e-commerce, se efectuó la realización de los Gráficos y Figuras utilizando el programa Excel (Aria \& Cuccurullo, 2017; Subramanyam, 1983; van Eck \& Waltman, 2014).

\section{Resultados y discusión}

En esta sección se describen y muestran gráficamente los resultados encontrados del análisis bibliométrico, destacando indicadores como los relacionados a la nube de palabras clave del e-commerce, la nube de palabras clave del B2B, los artículos publicados del e-commerce, el B2B, y el e-commerce con B2B entre 2010 al 2018, las publicaciones anuales del e-commerce y el B2B, el número de trabajos publicados en función del tipo de publicación, el mapa de producción científica por países, la colaboración global en las publicaciones analizadas, el número de artículos publicados contra el índice $\mathrm{G}$ de los autores por país, las publicaciones más relevantes en relación con las citas recibidas, las afiliaciones con más publicaciones y las fuentes más relevantes en las que se publica del e-commerce y el B2B.

El primer Gráfico de resultados relaciona la cantidad de artículos publicados de e-commerce, B2B, y el conjunto de artículos e-commerce y B2B del año 2010 al año 2018. 


\section{Gráfico 1}

Artículos publicados de e-commerce, B2B, y e-commerce y B2B del 2010-2018

e-commerce+b2b; 1,5

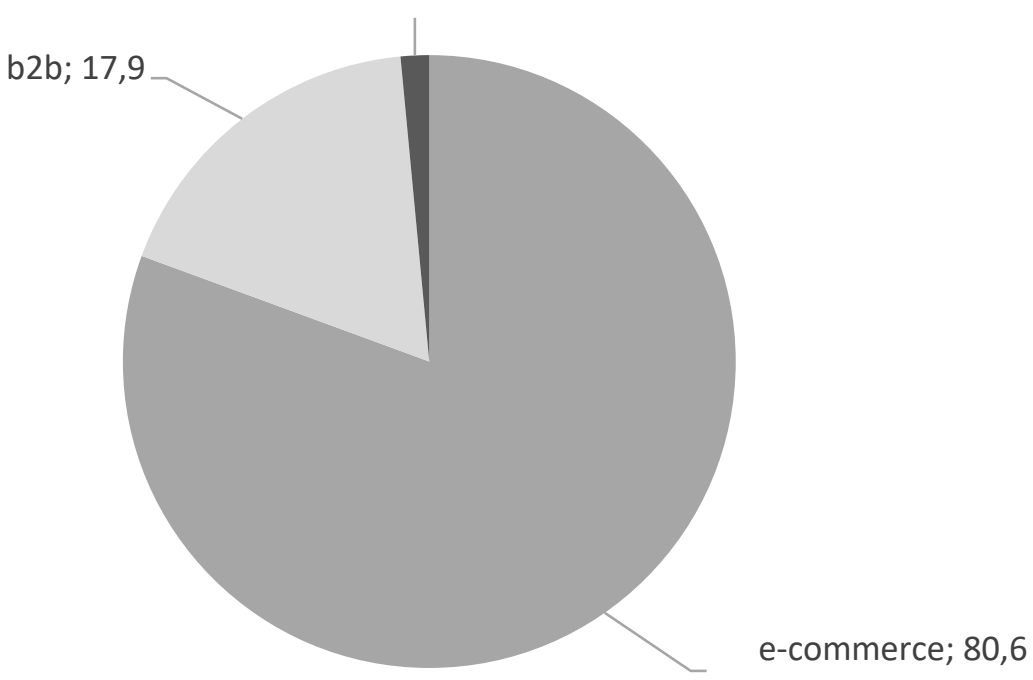

Fuente: Elaboración a partir de documentos de WoS y Scopus (2019).

Del Gráfico 1 se observa que el porcentaje de artículos que relacionan en conjunto al e-commerce y el B2B en el rango del año 2010 al año 2018, representa solo el 1.46\% del total, esto significa que existe una gran oportunidad para desarrollar futuras líneas de investigación científica relacionando con dichos temas. También se puede apreciar que el tema más grande y general de interés es el comercio electrónico, después el B2B y por último la conjunción de los dos temas anteriores.

Respecto a resultados relacionados con la mayor frecuencia de la nube de palabras clave relacionadas con el ecommerce se muestra la Figura 2.

Figura 2

Nube de palabras clave del e-commerce

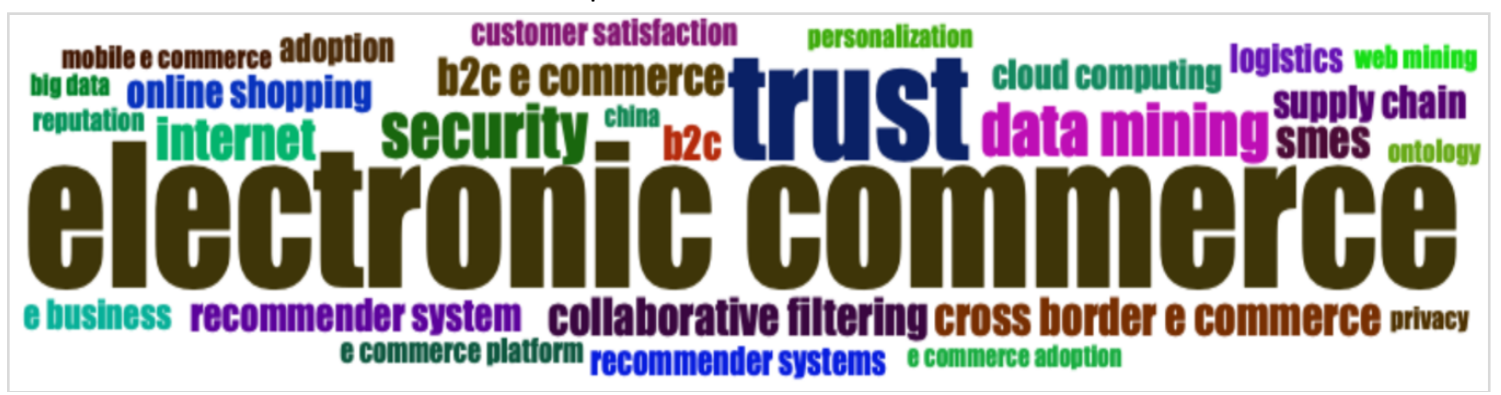

Fuente: Elaboración a partir de documentos de WoS y Scopus (2019).

En la Figura 2 se muestra la nube de palabras con mayor frecuencia en el título de los trabajos publicados del ecommerce, y se aprecia que los términos más frecuentes son: e-commerce, trust, data mining, security, e internet. Estas palabras representan los subtemas más representativos relacionados con el comercio electrónico. 
Respecto a los resultados relacionados con la mayor frecuencia de la nube de palabras de los artículos de B2B se aprecia la Figura 3.

Figura 3

Nube de palabras clave del B2B

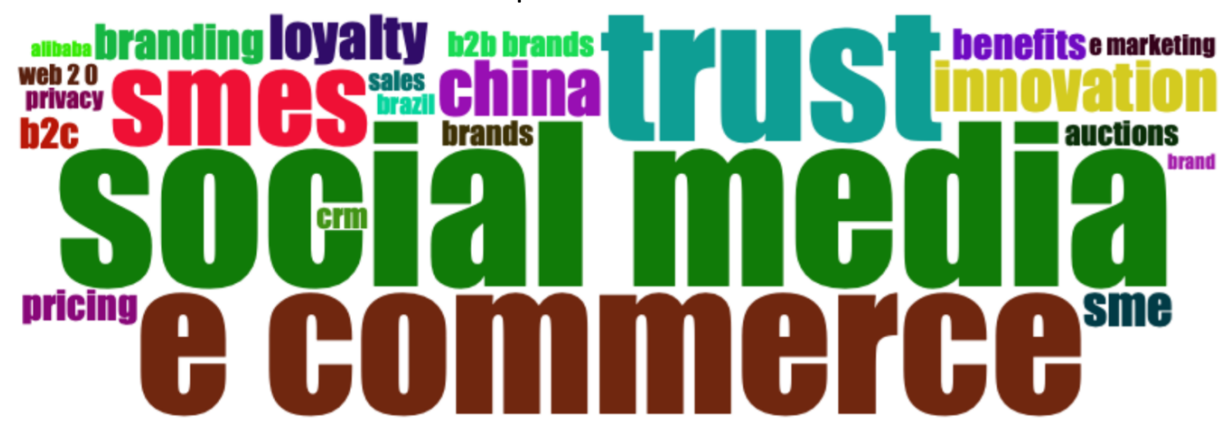

Fuente: Elaboración a partir de documentos de WoS y Scopus (2019).

De la Figura 3 relacionada con la palabra B2B y omitiendo el termino central de búsqueda e-commerce, se observa que palabras como social media, trust e innovation son las palabras más usuales. De los resultados anteriores también se puede apreciar que los autores que publican sobre B2B consideran al e-commerce un concepto clave, mientras que los autores que se enfocan al estudio del e-commerce no perciben al B2B como concepto recurrente, esto se debe a que existen otros modelos de comercio más recurrentes como B2C (Negocio a Consumidor), e-business y m-commerce. Destaca también la palabra smes relativa a que este tipo de estudios se realiza mucho más en pequeñas y medianas empresas (Pymes).

Respecto a los resultados relacionados a la cantidad de producción científica anual del 2010 al 2018 en la base de datos WoS y Scopus, se presentan en el Gráfico 2.

Gráfico 2

Publicaciones anuales de e-commerce y B2B de WoS y Scopus

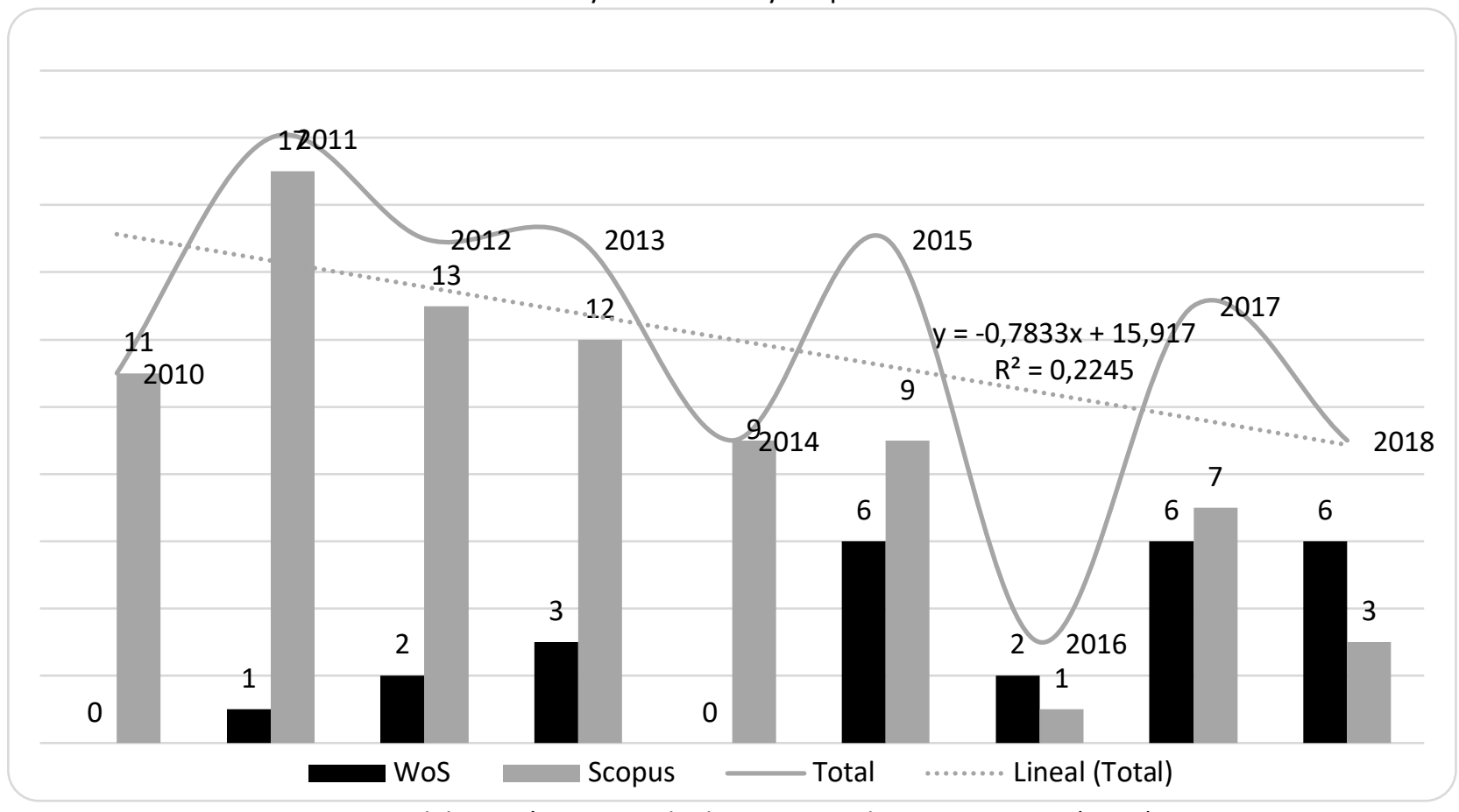

Fuente: Elaboración a partir de documentos de WoS y Scopus (2019) 
Del Gráfico 2 se aprecian dos situaciones principales, la primera es que la base de datos Scopus presenta una mayor cantidad de artículos de los temas en conjunto de e-commerce y B2B, la segunda es que el interés en el tema a decrecido del 2015 en adelante, en ambas bases de datos. Del punto anterior se puede apreciar también que en Scopus hubo una caída considerable de las publicaciones de estos temas desde el año 2011 hasta el año 2016, y posterior a ello se aprecia un ligero repunte en el número de publicaciones.

Por otra parte, en WoS se percibe de manera general una tendencia positiva e incremento de publicaciones de los temas desde el año 2010 al 2018, a excepción de dos inconsistencias en los años 2014 y 2016. En el 2016, en WoS y Scopus se percibe que hubo un ligero incremento de publicaciones registradas y la tendencia parece ser creciente pero lenta en los próximos años.

Por otra parte, el número de documentos por fuente o tipo de publicación se muestra en el Gráfico 3.

\section{Gráfico 3}

Número de trabajos en función del tipo de publicación

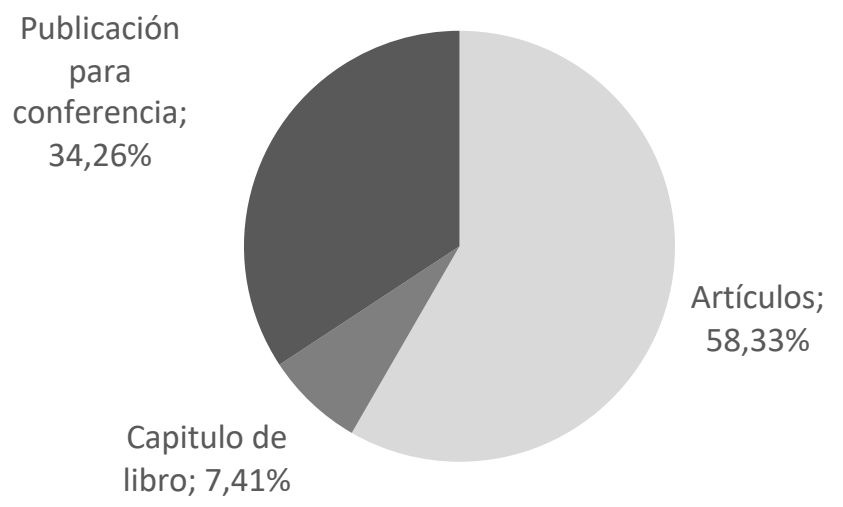

Fuente: Elaboración a partir de documentos de WoS y Scopus (2019).

En el Gráfico 3 se observa el total de producción científica de WoS y Scopus en relación al conjunto de documentos del e-commerce con B2B, se detectó que los artículos científicos fueron el medio de publicación más relevante con un $58.33 \%$, seguido de los artículos en conferencias con un $34.26 \%$ y posteriormente el $7.41 \%$ fue para la fuente de los capítulos de libro, esto representa un panorama global para ubicar las fuentes de información relevantes sobre los temas de e-commerce y B2B.

Con respecto a la producción científica con mayor frecuencia en los países del mundo, se presenta el Gráfico 4. 


\section{Gráfico 4}

Mapa de producción científica por país

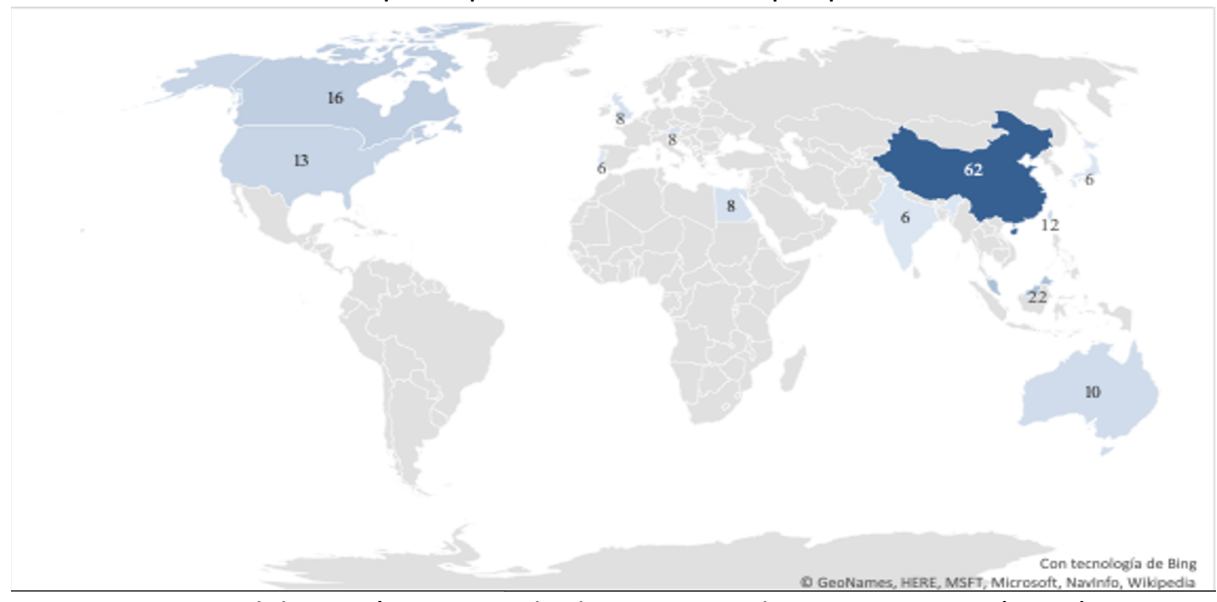

Fuente: Elaboración a partir de documentos de WoS y Scopus (2019).

Como se observa en el Gráfico 4, China resalta en producción científica en relación con B2B, siendo este país el que más artículos ha publicado relacionando el tema con e-commerce con un $28.97 \%$ del total de publicaciones, luego le sigue Malasia con un $10.28 \%$, enseguida Canadá con un $7.48 \%$, posteriormente Estados Unidos con $6.07 \%$, después Taiwán con $5.61 \%$ y más atrás Australia con $4.67 \%$. Del Gráfico anterior, resulta interesante observar que Brasil es el único país presente de Latinoamérica, esto a pesar de que el idioma requerido para publicar generalmente es el inglés; en este sentido, en el idioma español destaca España que cuenta con tres publicaciones.

Respecto a la colaboración global entre diferentes países se presenta la Tabla 1.

Tabla 1

Colaboración global en las publicaciones analizadas

\begin{tabular}{|c|c|c|c|}
\hline $\begin{array}{c}\text { Total de } \\
\text { colaboraciones }\end{array}$ & País de origen & País colaborador & Frecuencia \\
\hline \multirow[t]{4}{*}{12} & \multirow[t]{4}{*}{ Reino Unido } & Dinamarca & 2 \\
\hline & & Egipto & 1 \\
\hline & & Irak & 2 \\
\hline & & Estados Unidos & 1 \\
\hline \multirow[t]{4}{*}{12} & \multirow[t]{4}{*}{ Estados Unidos } & Dinamarca & 2 \\
\hline & & India & 1 \\
\hline & & Portugal & 2 \\
\hline & & Reino Unido & 1 \\
\hline \multirow[t]{4}{*}{9} & \multirow[t]{4}{*}{ China } & Reino Unido & 2 \\
\hline & & Estados Unidos & 3 \\
\hline & & Dinamarca & 1 \\
\hline & & Hong Kong & 1 \\
\hline \multirow[t]{4}{*}{8} & \multirow[t]{4}{*}{ Australia } & Malasia & 1 \\
\hline & & Taiwán & 3 \\
\hline & & Baréin & 1 \\
\hline & & Holanda & 1 \\
\hline \multirow[t]{2}{*}{7} & \multirow[t]{2}{*}{ Taiwán } & Canadá & 1 \\
\hline & & Filipinas & 2 \\
\hline \multirow[t]{4}{*}{6} & \multirow[t]{4}{*}{ Canadá } & China & 2 \\
\hline & & Chipre & 1 \\
\hline & & Taiwán & 1 \\
\hline & & Turquía & 1 \\
\hline
\end{tabular}




\begin{tabular}{clll}
\hline $\begin{array}{c}\text { Total de } \\
\text { colaboraciones }\end{array}$ & País de origen & País colaborador & Frecuencia \\
\hline 6 & Malasia & Jordania & 1 \\
\cline { 3 - 4 } & & Australia & 2 \\
\cline { 3 - 4 } & & Hong Kong & 1 \\
\hline 4 & Egipto & Irak & 2 \\
\cline { 2 - 4 } & & Reino Unido & 1 \\
\hline 3 & Portugal & Estados Unidos & 1 \\
\hline 2 & India & Estados Unidos & 1 \\
\hline 2 & Italia & Suiza & 2 \\
\hline 2 & Jordania & Malasia & 1 \\
\hline 2 & España & Reino Unido & 1 \\
\hline 1 & Bangladés & Ghana & 2 \\
\hline
\end{tabular}

Fuente: Elaboración a partir de documentos de WoS y Scopus (2019).

Como muestra la Tabla 1, Reino Unido y Estados Unidos son los países con mayor interacción y colaboración con otros países con 12 colaboraciones respectivamente, sin embargo, Estados Unidos es el cuarto país con mayor producción científica, solo por debajo de China con 49 publicaciones, de Malasia con 9 y de Canadá con 3 publicaciones.

Después, les sigue China con 9 colaboraciones y Australia con 8 seguidos por Taiwán con 7 y Canadá con seis colaboraciones. Por su parte, Malasia, a pesar de ser el segundo país que cuenta con más trabajos de publicación científica sobre los temas estudiados, es el quinto país en términos de colaboración, al igual que Canadá, con seis colaboraciones cada uno. Finalmente se observan otras colaboraciones menores de países como Egipto, Portugal, India, Italia, Jordania, España y Bangladés.

En cuanto al número de documentos publicados con respecto al índice $\mathrm{G}$ de los autores por país se presenta el Gráfico 5.

Gráfico 5

Número de documentos publicados contra índice $\mathrm{G}$ de los autores por país

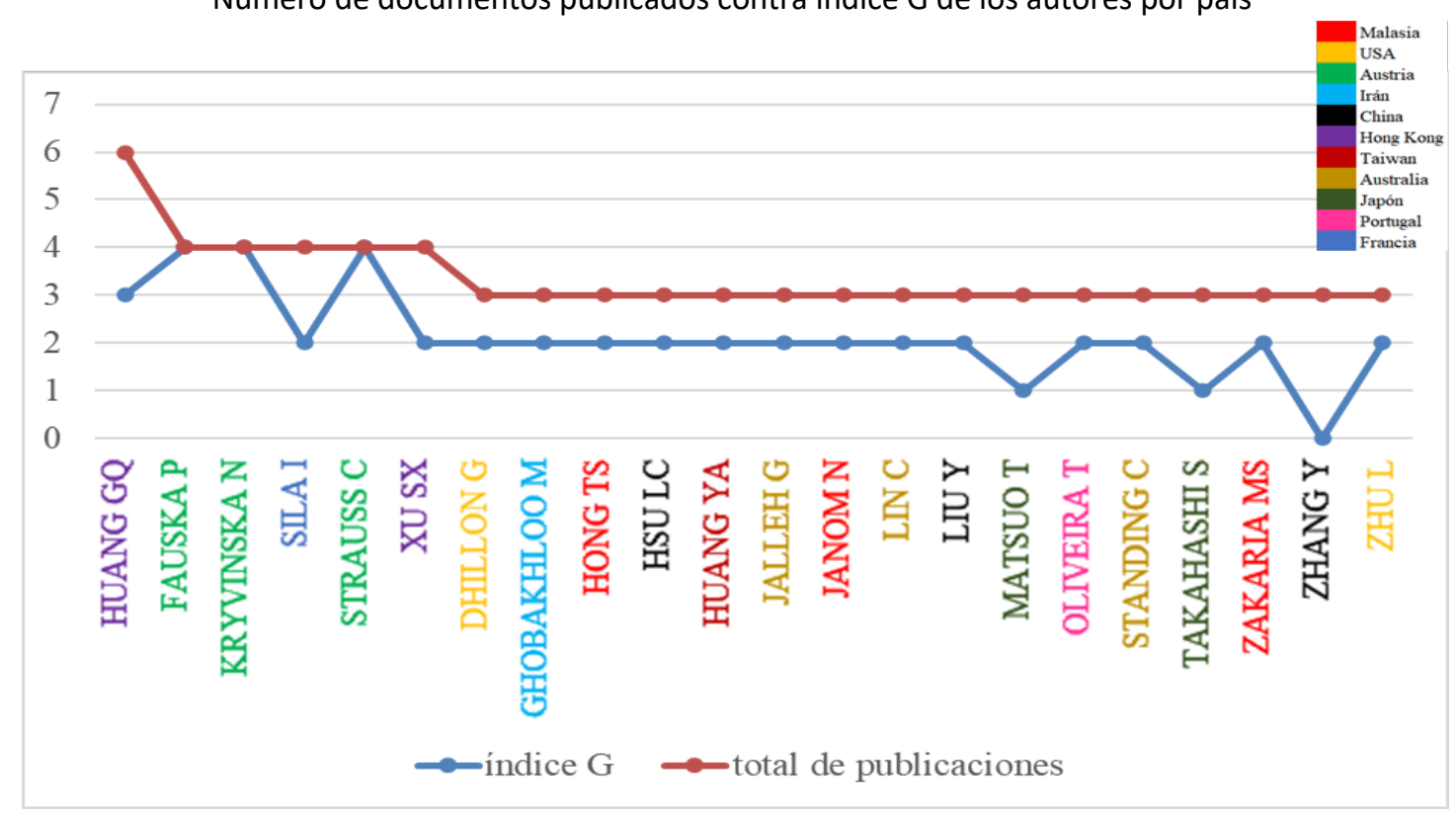

Fuente: Elaboración a partir de documentos de WoS y Scopus (2019). 
En cuanto a los documentos publicados del Gráfico 5 para evaluar a los autores que tenían mayor producción científica (línea roja del Gráfico), descartando a los que habían realizado menos de tres publicaciones en el acumulado de las dos bases de datos WoS y Scopus, y su relación con su reputación en el índice G (línea azul del Gráfico), el cual es un indicador que cuantifica la productividad bibliométrica basándose en el historial de publicaciones de los autores, calculado a partir de las citas recibidas por el número de publicaciones, este índice nos permite distinguir entre los autores con un índice $\mathrm{H}$ similar. Para el análisis se tomó en consideración la calificación más alta obtenida en las dos bases de datos, así como el país de publicación de sus artículos de acuerdo con el Gráfico 5. (Arencibia \& Carvajal, 2008; De Visscher, 2011; Egghe, 2006).

Del Gráfico anterior se observa que la cantidad de publicaciones no es proporcional al impacto que tuvieron sus respectivos autores, si bien, el máximo de artículos publicados fueron seis y el mínimo considerado fue de tres, son pocos los autores que obtuvieron un índice $G$ elevado por dichos trabajos, pues solo tres autores obtuvieron un índice de cuatro (Fauska, Kryvinska y Straus), todos ellos realizados en Austria, con cuatro publicaciones respectivamente. Por otro lado, se encuentra Huang de Hong Kong, el cual cuenta con un total de seis trabajos, siendo el mayor número de publicaciones registrado en del estudio, pero sus publicaciones han sido de menor impacto, alcanzando un tres en el índice G; mientras que Sila (2013), de Francia, también presenta algo similar, desarrollando cuatro publicaciones, pero evaluadas con un dos en el índice G.

En otra situación, Zhang con tres publicaciones realizadas en China, presenta un impacto nulo en el índice G. Lo anterior porque no está relacionado el impacto del autor con el número de publicaciones realizadas, como si es el caso, por ejemplo, el gran impacto de las autoras austriacas, siendo este un país con poca producción científica relacionadas a los temas analizados. Los autores más representativos en China, que es el país con mayor cantidad de publicaciones, el índice $\mathrm{G}$ de estos va de cero a dos, indicando que su impacto no ha sido grande.

En cuanto a las publicaciones más relevantes consideradas por las citas totales recibidas anualmente se presenta la Tabla 2.

Tabla 2

Publicaciones más relevantes en relación con las citas recibidas

\begin{tabular}{lcc}
\hline Autor y fuente (Revista) & $\begin{array}{c}\text { Citas } \\
\text { totales }\end{array}$ & $\begin{array}{c}\text { Citas totales } \\
\text { anuales }\end{array}$ \\
\hline Sila I. (2013), Electron Commer Res & 177 & 29.5 \\
Xu Sx. (2015), Transp Res Part B Methodol & 57 & 14.3 \\
Sila I. (2012), Ind Manage Data Sys & 56 & 8.0 \\
Chen Jv. (2013), Ind Manage Data Sys & 44 & 7.3 \\
Qu Wg. (2015), Inf Manage & 40 & 10.0 \\
Hsu Lc. (2013), Serv Ind J & 32 & 5.3 \\
Zhang M. (2017), Int J Prod Res & 31 & 15.5 \\
Xu Sx. (2017), Prod Oper Manage & 26 & 13.0 \\
Lip-Sam T. (2011), Int J Bus Soc & 24 & 3.0 \\
Oliveira T. (2015), J Global Inf Manage & 21 & 5.3 \\
\hline
\end{tabular}

Fuente: Elaboración a partir de documentos de WoS y Scopus (2019).

De la Tabla 2, se observa que autores con mayor frecuencia de citas totales también son generalmente los que cuentan con más citas anuales, tal es el caso de autores como Sila (2013) con 177 citas totales y 29.5 citas totales anuales (con un índice $\mathrm{G}$ de dos según Gráfico 5), Xu (2015) con 57 citas totales y 14.3 citas totales anuales ( con un índice $G$ de dos según Gráfico 5), y por otra parte, otros autores como Chen (2013), Qu (2015), Zhang (2017) y Lip-Sam(2011) (que no aparecen en el índice G del Gráfico 5), son los autores que le siguen a los mencionados. Por lo tanto, se puede deducir que el impacto de dichos autores debería ser mayor debido al impacto de sus publicaciones. 
Las afiliaciones o instituciones con más publicaciones, del tema del e-commerce y el B2B se presentan en la Tabla 3.

Tabla 3

Afiliaciones con más publicaciones

\begin{tabular}{llc}
\hline Afiliaciones & País y continente & $\begin{array}{c}\text { Número } \\
\text { de } \\
\text { The Unículos }\end{array}$ \\
University of Vienna & Hong Kong, Asia & 6 \\
Universiti Utara Malaysia & Austria, Europa & 6 \\
Carleton University & Malasia, Asia & 5 \\
Jinan University & Canadá, América & 4 \\
Shanghai University & China, Asia & 4 \\
Xi'an Jiaotong-Liverpool University & China, Asia & 4 \\
Cairo University & China, Asia & 4 \\
Edith Cowan University & Egipto, África & 3 \\
Girne American University & Australia, Oceanía & 3 \\
National Chi Nan University & Chipre, Europa & 3 \\
Universidade Nova De Lisboa & Taiwán, Asia & 3 \\
Universiti Putra Malaysia & Portugal, Europa & 3 \\
Virginia Commonwealth University & Malasia, Asia & 3 \\
Yamagata University & Estados Unidos, América & 3 \\
Zhejiang University & Japón, Asia & 3 \\
Univ Metodista Piracicaba Unimep & Brasil, América & 3 \\
\hline
\end{tabular}

Fuente: Elaboración a partir de documentos de WoS y Scopus (2019).

De la Tabla 3 se observa que la mayoría de los artículos presentes en el estudio han sido publicadas en universidades de países desarrollados, de lo anterior destacan las instituciones asiáticas. En primer lugar, se aprecia la Universidad de Hong Kong con seis publicaciones, al igual que la Universidad de Viena, institución donde colaboran las autoras más relevantes del tema según el índice G, y en tercer lugar, se encuentra la universidad Utara en Malasia con cinco publicaciones.

El resto de las instituciones relevantes con cuatro o menos publicaciones están ubicadas ya sea en China, como, Zhejiang University, Jinan University, Shanghai University, XI'AN Jiaotong-Liverpool University, en Canadá, como Carleton University, en Taiwán, como National Chi Nan University, en Egipto, como Cairo University, en Australia, como Edith Cowan University, en Chipre, como Girne American University, en Portugal, como Universidade Nova de Lisboa, en Malasia, como Universiti Putra Malaysia, en Estados Unidos, como Virginia Commonwealth University, en Japón, como Yamagata University y en Brasil, como Universidade Metodista Piracicaba Unimep.

En cuanto a las fuentes más relevantes y su índice $G$, relacionadas con el e-commerce y el B2B se presenta la Tabla 4.

Tabla 4

Fuentes más relevantes

\begin{tabular}{lcc}
\hline Fuente & Índice & $\begin{array}{c}\text { Publicaciones } \\
\text { totales }\end{array}$ \\
\hline Int. Conference on E-Business \& E-Government, Proceedings & G & 4 \\
Int. Journal of Information Science and Management & 1 & 3 \\
Advanced Materials Research & 1 & 2 \\
Industrial Management and Data Systems & 0 & 2 \\
Journal of Electronic Commerce in Organizations & 2 & 2 \\
\hline
\end{tabular}

Fuente: Elaboración a partir de documentos de WoS y Scopus (2019). 
De la Tabla 4 se aprecian las fuentes con mayor cantidad de publicaciones y su relevancia con el índice $\mathrm{G}$, en este sentido solo se consideraron las fuentes (conferencias o revistas de publicación científica) con dos o más publicaciones. De lo anterior, se obtuvo un total de tres fuentes con dos publicaciones, una con tres y una con cuatro publicaciones respectivamente.

Aunado a lo anterior, en la Tabla 4 y en el Gráfico 4 no se aprecia una relación entre la cantidad de artículos publicados y la relevancia de la fuente, ejemplos de ello son las publicaciones de la conferencia internacional de negocios electrónicos y gobierno electrónico de 2011 (ICEE2011) que cuentan con cuatro publicaciones pero mantienen un valor de uno en el índice G, así mismo se observa la revista Advanced Materials Research que cuenta con dos publicaciones pero con calificación de cero en el índice G. En conclusión, a través de este análisis se reveló que las revistas con mayor impacto según el índice $\mathrm{G}$ son Industrial Management and Data Systems y Journal of Electronic Commerce in Organizations, ambas con dos publicaciones y un índice $\mathrm{G}$ de dos.

\section{Conclusiones}

De los hallazgos encontrados en la sección de resultados se concluye de manera holística que el tema del ecommerce y el B2B se sigue desarrollando fuertemente en diferentes sectores organizacionales, sin embargo, hay una connotación mayor en las Pymes sin importar si estas pertenecen a las economías desarrolladas o no (Rowe, Truex \& Huynh, 2012). En este sentido, los países asiáticos y Estados Unidos son los más comprometidos con seguir estudiando el tema.

De los artículos publicados de e-commerce, B2B, y e-commerce y B2B del 2010 al 2018, el 80.64\% de estos se enfoca solo al comercio electrónico, mientras que un porcentaje menor estudia la conjunción del e-commerce y el B2B, lo que significa que estos últimos temas no han sido tan estudiados en la literatura general ni del ecommerce ni del B2B. Además, se encontró también que términos como la confianza, la seguridad y la minería de datos son aspectos clave en el comercio electrónico, mientras que términos como social media, la confianza y la innovación conforman los términos de mayor frecuencia de las investigaciones de la modalidad de Negocio a Negocio que se estudia principalmente en pequeñas y medianas empresas.

En cuanto a las publicaciones anuales de e-commerce y B2B en WoS y Scopus, se encontró que el tema visto de manera general en las dos bases de datos estudiadas mantiene una tendencia a la baja a pesar de tener algunos momentos con ciclos al alza. Los hallazgos encontrados podrían confirmar las afirmaciones de Hsu \& Chiang (2017), quienes también estudiaron los perfiles de investigación del e-commerce, y llegaron a la conclusión de que las modalidades o temáticas más relacionadas y estudiadas con el e-commerce no son aquellas relacionadas con el B2B, sino con otros modelos de comercio electrónico como el B2C, e-service, el m-commerce u otras modalidades que predominan mayormente en el tema (Kalia, Kaur \& Singh, 2018; Legner, 2008; Tian, Zhang \& Guan, 2013).

En cuanto al número de trabajos en función del tipo de publicación se encontró que en mayor proporción la fuente primaria son los artículos científicos con un 58.33\%, seguidamente de las publicaciones en conferencias con un 34.25\%. Respecto al mapa de producción científica por país se encontró que los países con más estudios del tema son China que cuenta con 64 publicaciones, luego le siguen Malasia, Canadá y Estados Unidos, este último con 13 publicaciones, predominando el continente asiático, sin embargo, en acuerdo con Hsu y Chiang (2017), los autores americanos mantienen una ligera ventaja en la citación y la relevancia de sus investigaciones, seguidamente de autores asiáticos.

Respecto a la colaboración global en las publicaciones analizadas, Reino Unido, Estados Unidos y China son los países que cuentan con mayores interacciones para la investigación con otros países. Del indicador anterior se puede afirmar que se requiere de un mayor trabajo colaborativo en la investigación de modelos de innovación 
abierta, lo que podría representar mayores beneficios para este tipo de investigaciones (Alvarez-Aros \& BernalTorres, 2017). Respecto al número de documentos publicados medidos por el índice $G$ de los autores por país, solo tres autores obtuvieron un índice de cuatro ( Fauska P., Kryvinska N. y Straus C.), todos ellos realizados en Austria, con cuatro publicaciones respectivamente.

Del punto anterior se concluyó que la calificación considerada por el índice G podría no ser tan precisa para representar la relevancia o el impacto de un autor en un análisis bibliométrico conforme a la afirmación de otros autores (Arencibia \& Carvajal, 2008; De Visscher, 2011; Díaz \& Sánchez, 2012; Egghe, 2006). Con respecto a las publicaciones más relevantes en relación con las citas recibidas se destacan autores como: Sila (2013) con 177 citas totales y 29.5 citas totales anuales (con un índice G de 2 según Grafica 5), Xu (2015) con 57 citas totales y 14.3 citas totales anuales (con un índice $\mathrm{G}$ de 2 según Gráfico 5 ).

Respecto a las afiliaciones o Universidades con más publicaciones sobre el tema, encontramos a la Universidad de Hong Kong y la Universidad de Viena, Austria, ambas con seis publicaciones. Esta última institución es en la que colaboran las autoras más relevantes del tema según el índice G. En tercer lugar, se encuentra la Universidad Utara, en Malasia, con cinco publicaciones. Respecto a las fuentes con mayor impacto conforme al índice $\mathrm{G}$ se pueden apreciar revistas como Industrial Management and Data Systems y Journal of Electronic Commerce in Organizations, ambas con dos publicaciones y un índice $\mathrm{G}$ de dos. Por último, se descubrió que la cantidad de artículos publicados no está relacionada con la relevancia de las revistas científicas donde estos se publican, y ocurre la misma situación con la relevancia de las revistas científicas en el análisis de los autores en la utilización del índice G.

Por todo lo anterior, se puede concluir que al explorar la relación entre el e-commerce y el B2B, los hallazgos permiten observar que la mayor contribución del tema es en estudios para las Pymes, además, también es necesario no perder de vista a otros modelos de comercialización como el m-commerce, y el B2C. Sin embargo, debemos reconocer que para examinar mejor dichos tipos de comercio es necesario estudiar otras modalidades de comercialización, como lo son el comercio electrónico móvil, el comercio electrónico con clientes, el comercio electrónico con gobierno, y representan líneas de investigación futura que complementarían significativamente el entono global de los nuevos métodos de comercialización.

\section{Referencias bibliográficas}

Alvarez-Aros, E. L., \& Bernal-Torres, C. A. (2017). Modelo de innovación abierta: énfasis en el potencial humano. Información tecnológica, 28(1), 65-76. doi/10.4067/S0718-07642017000100007

Alrawi, K. W., \& Sabry, K. A. (2009). E-commerce evolution: a Gulf region review. International Journal of Business Information Systems, 4(5), 509-526. doi:/10.1504/IJBIS.2009.025204

Arencibia, R., \& Carvajal, R. (2008). Los índices H, G y R: su uso para identificar autores líderes en el área de la comunicación durante el período 2001-2006. ACIMED, 17(4), 1-9.

De Visscher, A. (2011). What does the g-index really measure?. Journal of the American Society for Information Science and Technology, 62(11), 2290-2293. doi/10.1002/asi.21621

Díaz, I. A. L., \& Sánchez, Y. R. (2012). Análisis de los índices H, G y R en el sector agropecuario cubano a través de Scopus, 2005-2009. Anales de Documentación, 15 (1), 1-16. doi/10.6018/analesdoc.15.1.147641

Aria, M., \& Cuccurullo, C. (2017). Bibliometrix: An R-tool for comprehensive science mapping analysis. Journal of Informetrics, 11(4), 959-975. doi/10.1016/j.joi.2017.08.007 
Egghe, L. (2006). Theory and practise of the g-index. Scientometrics, 69(1), 131-152. doi/10.1007/s11192-0060144-7

Becerra, H. C., \& Fleitas, M. E. M. (2002). Bibliometría, Informetría, Cienciometría: su etimología y alcance conceptual. Revista Cubana de la ciencia.13(1), 1-15.

Blake, M. B. (2001). Innovations in software agent-based B2B technologies. In Workshop on Agent-Based Approaches to B2B at the Fifth International Conference on Autonomous Agents,1-7.

Brodie, M. (2000a). The B2B E-commerce revolution: convergence, chaos, and holistic computing. Information system engineering: state of the art and research themes. Springer, London, UK.

Brodie, M. (2000b). The B2B E-commerce revolution and its technology requirements. In National Science Foundation's Information and Data Management Workshop, Chicago, Illinois.

Burt, S., \& Sparks, L. (2003). E-commerce and the retail process: a review. Journal of Retailing and Consumer Services, 10(5), 275-286. doi/10.1016/S0969-6989(02)00062-0

Carlsson, H., Larsson, S., Svensson, L., \& Åström, F. (2017). Consumer credit behavior in the digital context: A bibliometric analysis and literature review. Journal of financial counseling and planning, 28(1), 76-94. doi/10.1891/1052-3073.28.1.76

Chandrasekar Subramaniam, M. J. S. (2002). A study of the value and impact of B2B e-commerce: the case of web-based procurement. International Journal of Electronic Commerce, 6(4), 19-40. doi/10.1080/10864415.2002.11044245

Chan, C. (2004). B2B e-commerce stages of growth: the strategic imperatives. In 37th Annual Hawaii International Conference on System Sciences. Proceedings of the IEEE, 1-10. doi/10.1109/HICSS.2004.1265560.

Damanpour, F., \& Damanpour, J. A. (2001). E-business e-commerce evolution: perspective and strategy. Managerial finance, 27(7), 16-33. doi/10.1108/03074350110767268

Dai, Q., \& Kauffman, R. J. (2002). B2B e-commerce revisited: Revolution or evolution? Guest editors' preface to the special section. Electronic Markets, 12(2), 64-66. doi/10.1080/10196780252844508

Donthu, N., Kumar, S., \& Pattnaik, D. (2020). Forty-five years of Journal of Business Research: A bibliometric analysis. Journal of Business Research, 109, 1-14. doi/10.1016/j.jbusres.2019.10.039

Eastin, M. S. (2002). Diffusion of e-commerce: an analysis of the adoption of four e-commerce activities. Telematics and informatics, 19(3), 251-267.doi/10.1016/S0736-5853(01)00005-3

Fensel, D., Ding, Y., Omelayenko, B., Schulten, E., Botquin, G., Brown, M., \& Flett, A. (2001). Product data integration in B2B e-commerce. IEEE Intelligent Systems, 16(4), 54-59.doi/ 10.1109/5254.941358

Fernández-Portillo, A., Sánchez-Escobedo, M. C., Jiménez-Naranjo, H. V., \& Hernández-Mogollón, R. (2015). The importance of innovation in e-commerce. Universia Business Review, 2015(47), 106-125.

Fernandez, L. M. V., Nicolas, C., \& Merigó, J. M. (2019). Industrial marketing research: a bibliometric analysis (1990-2015). Journal of Business \& Industrial Marketing.34(3),550-560. doi.org/10.1108/JBIM-07-20170167 
Fonseca, A. S. (2014). Fundamentos del e-commerce: Tu guía de comercio electrónico y negocios online. Alexandre Fonseca Lacomba.

Geng, S., Wang, Y., Zuo, J., Zhou, Z., Du, H., \& Mao, G. (2017). Building life cycle assessment research: A review by bibliometric analysis. Renewable and Sustainable Energy Reviews, 76, 176-184. doi/10.1016/j.rser.2017.03.068

Heng, M. S. (2003). Understanding electronic commerce from a historical perspective. Communications of the Association for Information Systems, 12(6), 1-17. doi/10.17705/1CAIS.01206

Hew, J. J. (2017). Hall of fame for mobile commerce and its applications: A bibliometric evaluation of a decade and a half (2000-2015). Telematics and Informatics, 34(1), 43-66. doi/10.1016/j.tele.2016.04.003

Jones, C., Motta, J., \& Alderete, M. V. (2016). Gestión estratégica de tecnologías de información y comunicación y adopción del comercio electrónico en Mipymes de Córdoba, Argentina. Estudios gerenciales, 32(138), 413.doi/10.1016/j.estger.2015.12.003

Hsu, C. L., \& Chiang, C. H. (2017). Electronic commerce research profiles: Comparing e-commerce and information systems journals. Pacific Asia Journal of the Association for Information Systems, 9(3), 49-66.

Kalia, P., Kaur, N., \& Singh, T. (2018). E-Commerce in India: evolution and revolution of online retail. Mobile Commerce: Concepts, Methodologies, Tools, and Applications. 736-758. doi/10.4018/978-1-5225-25998.ch036.

Kiron, D., Kane, G. C., Palmer, D., Phillips, A. N., \& Buckley, N. (2016). Aligning the organization for its digital future. MIT Sloan Management Review, 58(1).

Klopping, I. M., \& McKinney, E. (2004). Extending the technology acceptance model and the task-technology fit model to consumer e-commerce. Information Technology, Learning \& Performance Journal, 22(1), 35-48.

Kumar, B., Sharma, A., Vatavwala, S., \& Kumar, P. (2019). Digital mediation in business-to-business marketing: A bibliometric analysis. Industrial Marketing Management. https://doi.org/10.1016/j.indmarman.2019.10.002

Laudon, K. C., \& Traver, C. G. (2016). E-commerce: business, technology, society. Pearson,twelfth edition, (England) 1-41. http://repository.fue.edu.eg/xmlui/handle/123456789/4464

Legner, C. (2008). The evolution of B2B e-services from first generation e-commerce solutions to multichannel architectures. Journal of Electronic Commerce in Organizations, 6(2), 58-77.

Li, L., Sheng, X., \& Ma, L. (2018). E-business Research in China over the Last Two Decades: a Bibliometric Analysis of Projects Granted by National Social Science Fund of China. In Pacific Asia Conference on Information System PACIS, 1-28. https://aisel.aisnet.org/pacis2018/28

Marín-Idárraga, D. A., \& Cuartas-Marín, J. C. (2019). Relação entre inovação e desempenho: Impacto da intensidade competitiva e do slack organizacional. Revista de Administração de Empresas, 59(2), 95-107. doi/10.1590/s0034-759020190203

Martínez-López, F. J., Merigó, J. M., Valenzuela-Fernández, L., \& Nicolás, C. (2018). Fifty years of the European Journal of Marketing: a bibliometric analysis. European Journal of Marketing. 52(1/2), 439-468. doi/10.1108/EJM-11-2017-0853 
Merigó, J. M., \& Yang, J. B. (2017). A bibliometric analysis of operations research and management science. Omega, 73, 37-48. doi/10.1016/j.omega.2016.12.004

Moreno, M. B. G., Sánchez, J. J. N., Moreno, S. M. G., \& de Pablos Heredero, C. (2016). Evolución del negocio electrónico: un análisis bibliométrico de su adopción. Interciencia, 41(3), 148-153.

Mou, J., Cui, Y., \& Kurcz, K. (2019). Bibliometric and visualized analysis of research on major e-commerce journals using Citespace. Journal of Electronic Commerce Research, 20(4), 219-237.

Muhuri, P. K., Shukla, A. K., \& Abraham, A. (2019). Industry 4.0: A bibliometric analysis and detailed overview. Engineering applications of artificial intelligence, 78, 218-235. doi/10.1016/j.engappai.2018.11.007

Ngai, E. W., \& Wat, F. K. T. (2002). A literature review and classification of electronic commerce research. Information \& Management, 39(5), 415-429. doi/10.1016/S0378-7206(01)00107-0

Niranjanamurthy, M., Kavyashree, N., Jagannath, S., \& Chahar, D. (2013). Analysis of e-commerce and mcommerce: advantages, limitations and security issues. International Journal of Advanced Research in Computer and Communication Engineering, 2(6), 2360-2370.

Roig-Tierno, N., Gonzalez-Cruz, T. F., \& Llopis-Martinez, J. (2017). An overview of qualitative comparative analysis: A bibliometric analysis. Journal of Innovation \& Knowledge, 2(1), 15-23. doi/10.1016/j.jik.2016.12.002

Monroe, R. W., \& Barrett, P. T. (2019). The Evolving B2B E-Commerce and Supply Chain Management: A Chronological Mémoire. Journal of Business \& Management, 25(1). doi/10.6347/JBM.201903_25(1).0003.

Oliveira, R. V., Zhang, B., \& Zhang, L. (2007). Observing the evolution of Internet AS topology. ACM SIGCOMM Computer Communication Review, 37(4), 313-324. doi/ 10.1145/1282380.1282416

Rowe, F., Truex, D., \& Huynh, M. Q. (2012). An empirical study of determinants of e-commerce adoption in SMEs in Vietnam: An economy in transition. Journal of Global Information Management, 20(3), 23-54. doi/10.4018/jgim.2012070102.

Sila, I. (2013). Factors affecting the adoption of B2B e-commerce technologies. Electronic commerce research, 13(2), 199-236. doi/10.1007/s10660-013-9110-7

Subramanyam, K. (1983). Bibliometric studies of research collaboration: A review. Journal of information Science, 6(1), 33-38. doi/10.1177/016555158300600105

Thatcher, S. M., Foster, W., \& Zhu, L. (2006). B2B e-commerce adoption decisions in Taiwan: The interaction of cultural and other institutional factors. Electronic Commerce Research and Applications, 5(2), 92-104. doi/10.1016/j.elerap.2005.10.005

Tian, Z., Zhang, Z., \& Guan, X. (2013). A new evolution mechanism model for B2B e-commerce network. Journal of Electronic Commerce in Organizations, 11(2), 12-22. doi/10.4018/jeco.2013040102.

Tomak, K., \& Xia, M. (2002). Evolution of B2B marketplaces. Electronic Markets, 12(2), 84-91. doi/10.1080/10196780252844526

Tsai, H. H., \& Chiang, J. K. (2011). E-commerce research trend forecasting: A study of bibliometric methodology. International Journal of Digital Content Technology and its Applications, 5(1). 
Tuunainen, V. K. (1998). Opportunities of effective integration of EDI for small businesses in the automotive industry. Information \& Management, 34(6), 361-375. doi/10.1016/S0378-7206(98)00070-6

van Eck, N.J., \& Waltman L. (2014). Visualizing Bibliometric Networks. In: Ding Y., Rousseau R., Wolfram D. (eds) Measuring Scholarly Impact. Springer, Cham. doi/ 10.1007/978-3-319-10377-8_13

Valenzuela, L. M., Merigó, J. M., Johnston, W. J., Nicolas, C., \& Jaramillo, J. F. (2017). Thirty years of the Journal of Business \& Industrial Marketing: A bibliometric analysis. Journal of Business \& Industrial Marketing.32(1), 1-17. doi/10.1108/JBIM-04-2016-0079

Villa, E., Ruiz, L., Valencia, A., \& Picón, E. (2018). Electronic commerce: factors involved in its adoption from a bibliometric analysis. Journal of theoretical and applied electronic commerce research, 13(1), 3970.doi/10.4067/S0718-18762018000100104

Wang, J. J., Chen, H., Rogers, D. S., Ellram, L. M., \& Grawe, S. J. (2017). A bibliometric analysis of reverse logistics research (1992-2015) and opportunities for future research. International Journal of Physical Distribution \& Logistics Management.47(8), 666-687. doi/10.1108/IJPDLM-10-2016-0299

Wigand, R. T. (1997). Electronic commerce: Definition, theory, and context. The information society, 13(1), 1-16. doi/10.1080/01972249712924.

Wilkinson, I. F. (2006). The evolution of an evolutionary perspective on B2B business. Journal of Business \& Industrial Marketing, 21(7), 458-465. doi/10.1108/08858620610708957

Williams, M. L., \& Frolick, M. N. (2001). The evolution of EDI for competitive advantage: The FedEx case. Information Systems Management, 18(2), 47-53.doi/10.1201/1078/43195.18.2.20010301/31277.8

Xiao, S., \& Dong, M. (2015). Hidden semi-Markov model-based reputation management system for online to offline (O2O) e-commerce markets. Decision Support Systems, 77, 87-99. doi/10.1016/j.dss.2015.05.013

Zadykowicz, A., Gębarowski, M., \& Siemieniako, D. (2019). Experience Marketing and Business to Business Interactions in the Light of Bibliometric Analysis. Przedsiębiorczość i Zarzqqdzanie, 20(6, cz. 2 Zmiany w myśleniu marketingowym), 259-275. doi/bwmeta1.element.ekon-element-000171560321

Zhu, L., Li, L., Zhang, Y., \& Shi, Q. (2017). Strategic IT investment in B2B e-commerce platform on user loyalty: considering variable investment cost. International Journal of Networking and Virtual Organisations, 17(23), 290-313. doi/10.1504/IJNVO.2017.085517

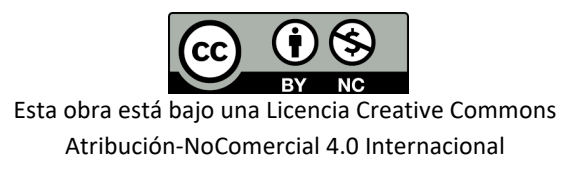

